\title{
MicroRNA-Based Cancer Mortality Risk Scoring System and hTERT Expression in Early-Stage Oral Squamous Cell Carcinoma
}

\author{
Angela J. Yoon $\mathbb{D}^{1},{ }^{1}$ Regina M. Santella, ${ }^{2}$ Shuang Wang, ${ }^{2}$ David I. Kutler, ${ }^{3}$ \\ Richard D. Carvajal, ${ }^{1}$ Elizabeth Philipone, ${ }^{1}$ Tian Wang, ${ }^{2}$ Scott M. Peters, ${ }^{1}$ Claire R. Stewart, ${ }^{3}$ \\ Fatemeh Momen-Heravi, ${ }^{1}$ Scott Troob, ${ }^{1}$ Matt Levin, ${ }^{4}$ Zohreh AkhavanAghdam, ${ }^{4}$ \\ Austin J. Shackelford, ${ }^{1}$ Carleigh R. Canterbury, ${ }^{1}$ Masataka Shimonosono, ${ }^{1}$ \\ Brenda Y. Hernandez, ${ }^{5}$ Bradley D. McDowell, ${ }^{6}$ and Hiroshi Nakagawa ${ }^{1}$ \\ ${ }^{1}$ Columbia University Irving Medical Center, New York, NY, USA \\ ${ }^{2}$ Columbia University Mailman School of Public Health, New York, NY, USA \\ ${ }^{3}$ Weill Cornell Medicine, New York, NY, USA \\ ${ }^{4}$ Cell IDx, San Diego, CA, USA \\ ${ }^{5}$ Hawaii Tumor Registry, University of Hawaii Cancer Center, Honolulu, HI, USA \\ ${ }^{6}$ Holden Comprehensive Cancer Center, University of Iowa, Iowa City, IA, USA
}

Correspondence should be addressed to Angela J. Yoon; ajk55@columbia.edu

Received 2 July 2020; Revised 1 December 2020; Accepted 14 December 2020; Published 15 January 2021

Academic Editor: Dali Zheng

Copyright ( 92021 Angela J. Yoon et al. This is an open access article distributed under the Creative Commons Attribution License, which permits unrestricted use, distribution, and reproduction in any medium, provided the original work is properly cited.

We have previously constructed a novel microRNA (miRNA)-based prognostic model and cancer-specific mortality risk score formula to predict survival outcome in oral squamous cell carcinoma (OSCC) patients who are already categorized into "earlystage" by the TNM staging system. A total of 836 early-stage OSCC patients were assigned the mortality risk scores. We evaluated the efficacy of various treatment regimens in terms of survival benefit compared to surgery only in patients stratified into high (risk score $\geq 0$ ) versus low (risk score $<0$ ) mortality risk categories. For the high-risk group, surgery with neck dissection significantly improved the 5 -year survival to $75 \%$ from $46 \%$ with surgery only $(p<0.001)$; a Cox proportional hazard model on time-to-death demonstrated a hazard ratio of 0.37 for surgery with neck dissection (95\% CI: $0.2-0.6 ; p=0.0005)$. For the low-risk group, surgery only was the treatment of choice associated with 5-year survival benefit. Regardless of treatment selected, those with risk score $\geq 2$ may benefit from additional therapy to prevent cancer relapse. We also identified hTERT (human telomerase reverse transcriptase) as a gene target common to the prognostic miRNAs. There was 22-fold increase in the hTERT expression level in patients with risk score $\geq 2$ compared to healthy controls $(p<0.0005)$. Overexpression of hTERT was also observed in the patientderived OSCC organoid compared to that of normal organoid. The DNA cancer vaccine that targets hTERT-expressing cells currently undergoing rigorous clinical evaluation for other tumors can be repurposed to prevent cancer recurrence in these highrisk early-stage oral cancer patients.

\section{Introduction}

An estimated 49,000 people in the United States are diagnosed with oral squamous cell carcinoma (OSCC) each year $[1,2]$. Leading etiologic factors include tobacco and alcohol [1-5]. While $80 \%$ of oropharyngeal cancers are related to high-risk human papillomavirus (HPV types 16 and 18), the incidence of high-risk HPV-related oral cancer is relatively low, estimated to be $\sim 4 \%[4,5]$. Despite advances in cancer diagnosis and treatment, the overall 5-year survival rate for OSCC remains the lowest among malignancies and, in fact, has been $<50 \%$ for the last three decades [1-5]. Among 
newly diagnosed oral cancer cases, $\sim 80 \%$ are in the tumornode-metastasis (TNM) Stage I/II without regional lymph node involvement or distant metastasis [3]. Hence, a window of opportunity exists in which accurate prognostication and subsequent decisions for appropriate treatment could dramatically improve 5 -year survival of patients with this deadly disease.

While the TNM stage is considered the key prognostic determinant in oral cancer [6], it is incapable of delineating individual risk for patients within the same TNM stage strata. To address these critical gaps, we have constructed a microRNA (miRNA)-based prognostic model and cancerspecific mortality risk score formula using a pool of earlystage oral cancer patients who had surgery only as initial treatment [7]. miRNAs are small, 18-24 nucleotide long, noncoding RNA molecules that regulate the expression of targeted genes either by facilitating mRNA degradation or by repressing translation $[8,9]$. One miRNA is capable of binding over 100 different mRNAs with different binding efficiencies and plays a crucial role in their posttranscriptional regulation [8-12]. miRNAs control cell growth, apoptosis, and differentiation, and various types of cancer have demonstrated distinct miRNA expression profiles [8-12]. Thus far, a number of miRNAs associated with clinical outcomes have been reported for lung, breast, gastric, and pancreatic cancers, as well as OSCC/head and neck carcinomas [12-14].

In our miRNA-based prognostic model, we included pertinent clinicodemographic covariates [7]. Therefore, the final prognostic model was built based on both expression of the miRNAs (miRNA-127-3p, 4736, 655-3p) and clinicodemographic covariates (TNM Stage I vs II, histologic grade well vs moderate/poor), which allowed for multiple risk factors to be used systematically and reproducibly to maximize the prognostic power. The prognostic model was subject to rigorous internal validation, followed by external validation, which included patient populations from Pennsylvania, Iowa, and Hawaii [7]. The performance of the model, in terms of its discriminatory power to differentiate between high and low cancer-specific mortality risks, was meticulously verified. The area under the curve (AUC) of the receiver operating characteristic (ROC) curve with the miRNA-based 5-plex marker panel was $0.83(p<0.001), 0.87$ $(p<0.001)$, and $0.81(p<0.001)$ in internal test, internal validation, and external validation cohorts, respectively, demonstrating uniformly significant prognostic value [7]. The clinical prognostic indicators alone, including TNM stage and histologic grading, had an AUC of $0.67(p<0.001)$, demonstrating that the 5-plex prognostic marker significantly increase the prognostic power [7].

From the final prognostic model, we constructed a robust mortality risk score formula. This personalized formula consisted of the patient's miRNA expression levels and prognostic covariates weighed by their regression coefficient. The main purpose of the risk score formula is for clinicians to easily translate miRNA levels obtained from the clinical laboratory, along with known clinicodemographic variables, into the patient's risk scores, which will serve as a practical method to assess patient-specific mortality risk in the clinical setting. The risk score from the 5-plex marker panel consisting of miRNAs-127-3p, 4736, 655-3p, TNM stage, and histologic grading stratified patients initially into high $(\geq 0)$ vs low $(<0)$ risk categories, and then sub-risk stratified into finer risk categories [highest (risk score $\geq 2$ ) vs moderately high (risk score 1-2) vs moderately low (risk score $0-1$ ) vs low (risk score $<0)]$. Compared to the low-risk strata $(<0)$, the highest-risk strata $(\geq 2)$ had 23 -fold increased mortality risk (hazard ratio of $23,95 \%$ confidence interval 13-42), with a median time-to-recurrence of 6 months and time-to-death of 11 months (versus $>60$ months for both outcomes among low-risk patients; $p<0.001)$.

In this study, we evaluated the efficacy of various treatment regimens in patients stratified into high vs. low mortality risk categories by the miRNA-based prognostic risk score formula. In specific, we assessed the median timeto-recurrence, the median time-to-death, and 5-year survival rate in patients who received surgery only $(\mathrm{S})$ vs. surgery with neck dissection $(S+\mathrm{ND})$ vs. surgery with irradiation $(S+\mathrm{IR})$ vs. surgery with ND and IR $(S+\mathrm{ND} / \mathrm{IR})$ as the initial surgical treatment and compared the survival benefit associated with each treatment.

\section{Materials and Methods}

2.1. Subjects and Study Design. Following approval from the Institutional Review Board (IRB), 836 early-stage OSCC patients, $\geq 18$ years old, newly diagnosed with primary OSCC, and with a minimum of 5-year clinical outcomes information were identified from Columbia University Irving Medical Center, Weill Cornell Medicine, University of Hawaii Cancer Research Center, the Iowa Cancer Registry at the University of Iowa, and the Eastern Division of Cooperative Human Tissue Network (CHTN). Subjects who were found to have occult lymph node metastasis following initial surgery were excluded. Subjects with the Eastern Cooperative Oncology Group (ECOG) performance-status score of 0 (no symptoms) or 1 (mild symptoms) were included.

The following clinicodemographic information was obtained from the electronic clinical record: age at diagnosis, gender, race/ethnicity (white non-Hispanic, white Hispanic, black non-Hispanic, black Hispanic, Asian), TNM stage (I vs II), histologic tumor grade (well vs moderate-to-poorly differentiated), treatment received (surgery with or without neck dissection, irradiation, or both neck dissection and irradiation), tobacco use (never/former vs current), and alcohol abuse (4 or more drinks on any day or 8 or more drinks per week; never/former vs current). Time of initial surgical treatment until cancer recurrence and cancer-specific death were also noted.

For each subject, archived formalin-fixed paraffin-embedded (FFPE) tissue blocks were retrieved. In case the subject had recurrent and/or second primary OSCC, the initial OSCC surgical tissue sample was utilized for the analysis. Ten $10-\mu \mathrm{m}$ sections were obtained from archived FFPE tumor tissue samples for all subjects. For each sample, a representative section was stained with $\mathrm{H} \& \mathrm{E}$ and reviewed by a pathologist to identify regions containing $>90 \%$ 
malignant epithelial cells for microdissection as previously described [7]. Total RNA was isolated from tissues using RNeasy FFPE kits (Qiagen Inc., Valencia, CA) following the manufacturer's protocol, yield was quantitated by Nanodrop, and samples were stored at $-80^{\circ} \mathrm{C}$.

\subsection{MicroRNA Expression Assessment by Quantitative Real-} Time PCR (qRT-PCR). The prognostic miRNA-127-3p, 4736, 655-3p expression levels were quantified using the miScript II Rt kit (Qiagen) as described previously [7]. Briefly, $9 \mu \mathrm{L}$ of isolated RNA was added to the cDNA master mix, composed of 5x miScript HiSpec Buffer, 10x miScript Nucleics Mix, miScript Reverse Transcriptase Mix, and water, to a total volume of $20 \mu \mathrm{L}$. The cDNA was incubated at $37^{\circ} \mathrm{C}$ for $60 \mathrm{~min}$, followed by $5 \mathrm{~min}$ incubation at $95^{\circ} \mathrm{C}$, and then diluted 11 times. For amplification reactions, the miScript miRNA PCR Custom Array with a miScript SYBR Green PCR kit (Qiagen) was used in a 7300 qPCR system (Applied Biosystems Inc., Beverly, MA), following the cycling conditions recommended by the supplier $(15 \mathrm{~min}$ at $95^{\circ} \mathrm{C}$, followed by 40 cycles of $15 \mathrm{~s}$ at $94^{\circ} \mathrm{C}, 30 \mathrm{~s}$ at $55^{\circ} \mathrm{C}$, and $30 \mathrm{~s}$ at $70^{\circ} \mathrm{C}$ ). The coefficient of variation was calculated and values $<5 \%$ were considered acceptable. Test samples were assayed in duplicate with the laboratory blinded to survival status and with 5\% duplication after relabeling. Data was analyzed to determine the threshold cycle $(\mathrm{Ct})$. The endogenous control RUN6-6p was used to normalize the relative expression of target miRNAs $(\Delta \mathrm{Ct})$. The samples with undetermined $\mathrm{Ct}$ value for the control were excluded from analysis. Those with an undetermined $\mathrm{Ct}$ for specific miRNAs were assigned a value of 39.99.

2.3. Mortality Risk Score Calculation. Using the miRNAbased mortality risk score formula, the risk score was calculated for all subjects as previously described [7]. The mortality risk scores were obtained by summing the expression values of the selected miRNAs and covariates weighted by the regression coefficients obtained from the multivariate Cox regression analyses. Mortality risk score $=(-0.7 x$ expression value of miRNA-127-3p $)+(-0.3 x$ expression value of miRNA-4736 $)+(0.1 x$ expression value of miRNA-655-3p $)+(0.9 \times 0$ for TNM Stage I; 1 for TNM Stage II $)+(0.4 \times 0$ for well-differentiated; 1 for moderately/ poorly differentiated), in which the miRNA expression level is the $\Delta \mathrm{Ct}$ value of each miRNA. Based on the individual mortality risk score, the patients were first stratified into high $(\geq 0)$ vs. low $(<0)$ mortality risk groups and then further stratified into highest $(\geq 2)$, moderately high $(1$ to $<2)$, moderately low $(0$ to $<1)$, and low $(<0)$ risk groups.

2.4. Prognostic miRNA Functional Analysis. Network visualization and functional analysis were previously performed using Cytoscape v3.2.0 to identify potential gene targets of miRNA-127-3p, 4736, 655-3p [7]. We used the gene targets from the previous study to identify the intersection among genes that control cancer recurrence and aggressiveness.
Venn diagram was constructed to visualize gene targets common to three miRNAs.

2.5. Statistical Analysis. We calculated the individual mortality risk scores for all patients in the four treatment groups using our miRNA-based mortality risk score formula. Based on these mortality risk scores, the patients were stratified into high $(\geq 0)$ vs. low $(<0)$ mortality risk groups. For each risk group, the median time-to-death and the median timeto-recurrence as well as the 5-year survival rate were assessed. The hazard ratios (HR) of the treatments $S+\mathrm{ND}$, $S+I R$, and $S+N D /$ IR were computed over S. The KaplanMeier curves were generated for the high- and low-risk strata of the four treatment groups. Statistical analyses were conducted using $R$.

\section{Results}

3.1. Subject Characteristics. The demographic and clinicopathologic characteristics of each treatment category are shown in Table 1 (total $n=836$ ). The treatment groups include surgery only $(S ; n=551)$, surgery with neck dissection $(S+\mathrm{ND} ; n=164)$, surgery with radiotherapy $(S+\mathrm{IR} ; n=76)$, and surgery with neck dissection and irradiation $(S+\mathrm{ND} / \mathrm{IR} ; n=45)$. Compared to $\mathrm{S}$ group, significantly more patients with higher TNM stage (Stage II) and histologic tumor grade (moderately/poorly differentiated carcinoma) received treatment in addition to surgery.

3.2. Mortality Risk Score-Based Stratification. The miRNAbased mortality risk score formula was utilized to calculate the risk score for all patients. Within each treatment group, the patients were stratified into high-risk $(\geq 0)$ vs low-risk $(<0)$. The median time-to-recurrence and time-to-death as well as 5-year survival rates were calculated for high vs. low-risk strata in each treatment group (Table 2). Within the high-risk strata, $S+\mathrm{ND}$ significantly improved the 5-year survival (75\%) over S (46\%). Compared to $S+\mathrm{ND}, S+\mathrm{IR}$ was not associated with survival benefit (44\% 5-year survival rate for $S+$ IR and $62 \%$ for $S+$ ND/IR) as shown in Figure 1(a). The HR of $S+\mathrm{ND}$ over $S$ was 0.37 (95\% CI: $0.2-0.6)$ with a $p$-value of 0.0005 , indicating survival benefit with $S+\mathrm{ND}$ for high-risk patients.

In the low-risk strata, $\mathrm{S}$ was associated with the highest 5-year survival rate compared to other treatment modalities (Table 2). $S+$ ND that demonstrated significant survival benefit in the high-risk strata was associated with lower 5-year survival of $74 \%$ compared to S (5-year survival rate of $89 \%$ ) in the low-risk strata. Similarly, $S+$ IR and $S+\mathrm{ND} / \mathrm{IR}$ did not offer survival benefit over $\mathrm{S}$ for low-risk patients. The HR of $S+$ ND over S was 2.6 (95\% CI: 1.6-4.2), for $S+$ IR 6.5 (95\% CI: 3.9-10.8), and for $S+\mathrm{ND} / \mathrm{IR} 3.4$ (95\% CI: $1.7-6.7$ ) all with $p$-values $\leq 0.0001$. Thus, for the low mortality risk strata, surgery only was the best treatment option (Figure 1(b)). 
TABLE 1: Clinical and histopathologic characteristics of the early-stage oral squamous cell carcinoma patients included in this study.

\begin{tabular}{|c|c|c|c|c|}
\hline & S only & $S+\mathrm{ND}$ & $S+\mathrm{IR}$ & $S+\mathrm{ND} / \mathrm{IR}$ \\
\hline Patients & $n=551$ & $n=164$ & $n=76$ & $n=45$ \\
\hline \multicolumn{5}{|l|}{ Age } \\
\hline Mean (range) & $66(21-97)$ & $61(25-87)$ & $65(24-90)$ & $58(35-80)$ \\
\hline \multicolumn{5}{|l|}{ Gender } \\
\hline Female & $218(40 \%)$ & $84(51 \%)$ & $24(32 \%)$ & $21(47 \%)$ \\
\hline Male & $333(60 \%)$ & $80(49 \%)$ & $52(68 \%)$ & $24(53 \%)$ \\
\hline \multicolumn{5}{|l|}{ Race/Ethnicity } \\
\hline White non-Hispanic & $383(70 \%)$ & $92(56 \%)$ & $30(39 \%)$ & $22(49 \%)$ \\
\hline White hispanic & $80(15 \%)$ & $15(9 \%)$ & $9(12 \%)$ & $9(20 \%)$ \\
\hline Black non-Hispanic & $22(4 \%)$ & $4(2 \%)$ & $1(1 \%)$ & $7(6 \%)$ \\
\hline Black hispanic & $5(1 \%)$ & $0(0 \%)$ & $0(0 \%)$ & $0(0 \%)$ \\
\hline Asian & $52(9 \%)$ & $34(21 \%)$ & $27(36 \%)$ & $5(11 \%)$ \\
\hline Unknown & $9(2 \%)$ & $19(12 \%)$ & $9(12 \%)$ & $2(4 \%)$ \\
\hline \multicolumn{5}{|l|}{ TNM Stage } \\
\hline Stage I & $428(78 \%)$ & $81(49 \%)$ & $40(53 \%)$ & $17(38 \%)$ \\
\hline Stage II & $123(22 \%)$ & $83(51 \%)$ & $36(47 \%)$ & $28(62 \%)$ \\
\hline \multicolumn{5}{|l|}{ Histologic grading } \\
\hline Well-differentiated & $291(53 \%)$ & $76(46 \%)$ & $29(38 \%)$ & $8(18 \%)$ \\
\hline Moderately/poorly differentiated & $260(47 \%)$ & $88(54 \%)$ & $47(62 \%)$ & $37(82 \%)$ \\
\hline \multicolumn{5}{|l|}{ Smoking Status } \\
\hline Never & $163(30 \%)$ & $64(39 \%)$ & $2(3 \%)$ & $17(38 \%)$ \\
\hline Past & $72(13 \%)$ & $39(24 \%)$ & $9(12 \%)$ & $7(16 \%)$ \\
\hline Current & $54(48 \%)$ & $12(7 \%)$ & $5(7 \%)$ & $6(13 \%)$ \\
\hline Unknown & $262(48 \%)$ & $49(30 \%)$ & $60(79 \%)$ & $15(33 \%)$ \\
\hline \multicolumn{5}{|l|}{ Alcohol Abuse } \\
\hline Never & $201(36 \%)$ & $80(49 \%)$ & $4(5 \%)$ & $22(49 \%)$ \\
\hline Past & $32(6 \%)$ & $14(9 \%)$ & $6(8 \%)$ & $0(0 \%)$ \\
\hline Current & $56(10 \%)$ & $21(13 \%)$ & $6(8 \%)$ & $8(18 \%)$ \\
\hline Unknown & $262(48 \%)$ & $49(30 \%)$ & $60(79 \%)$ & $15(33 \%)$ \\
\hline
\end{tabular}

TABLE 2: The efficacy of various treatment regimens in oral squamous cell carcinoma patients stratified into high (risk score $\geq 0$ ) versus lowrisk (risk score $<0$ ) using the microRNA-based prognostic model; surgery $(S)$ only, surgery with neck dissection $(S+N D)$, surgery with irradiation $(S+I R)$, and surgery with neck dissection and irradiation $(S+N D / I R)$.

\begin{tabular}{|c|c|c|c|c|c|}
\hline Treatment & Risk category & Median time-to-recurrence & Median time-to-death & Cancer death (\%) & 5-year survival (\%) \\
\hline \multirow{2}{*}{$S$} & High risk $(\geq 0)$ & 26 (months) & 50 (months) & 54 & 46 \\
\hline & Low risk $(<0)$ & $\geq 60$ & $\geq 60$ & 11 & 89 \\
\hline \multirow{2}{*}{$S+\mathrm{ND}$} & High risk & $\geq 60$ & $\geq 60$ & 25 & $75^{*}$ \\
\hline & Low risk & $\geq 60$ & $\geq 60$ & 26 & $74^{*}$ \\
\hline \multirow{2}{*}{$S+\mathrm{IR}$} & High risk & 41 & 43 & 56 & 44 \\
\hline & Low risk & 33 & $\geq 60$ & 49 & $51^{*}$ \\
\hline \multirow{2}{*}{$S+\mathrm{ND} / \mathrm{IR}$} & High risk & 7 & $\geq 60$ & 38 & 62 \\
\hline & Low risk & $\geq 60$ & $\geq 60$ & 34 & $66^{*}$ \\
\hline
\end{tabular}

${ }^{*} p \leq 0.05$ when compared with the same risk strata of S only.

3.3. Sub-Risk Strata Analysis of $S$ versus $S+N D$. For the patients who were treated with $S$ or $S+\mathrm{ND}$, we compared the median time-to-recurrence, the median time-to-death, and 5year survival rate by substratifying risk categories to highest $(\geq 2)$, moderately high $(<2-1)$, moderately low $(<1-0)$, and low $(<0)$ as shown in Table 3 . For the patients in the moderately high and moderately low substrata, the 5-year survival rates were $29 \%$ and $58 \%$, respectively, with S. However, if these patients received $S+$ ND instead, the 5-year survival rate increased to $100 \%$. The patients with risk score $\geq 2$ who had S as initial treatment demonstrated significantly shorter median time-torecurrence (6 months), median time-to-death (11 months), and the 5-year survival rate of $6 \%$; with $S+\mathrm{ND}$, the survival rate improved to $68 \%$. For the patients in the low-risk substrata (risk score $<0$ ), $S$ consistently demonstrated survival benefit over $S+$ ND. The clinical risk categories, high (risk score $\geq 2$ ) vs. moderate (risk score between 0 and $<1$ ) vs. low-risk (risk score $<0)$, can be used as a guideline to select initial surgical treatment and also to identify high-risk patients (risk score $\geq 2$ ) who may need therapy additional to surgery to optimize survival.

3.4. Functional Analysis of Prognostic miRNAs. We had previous identified common gene targets of miRNA-127-3p, 


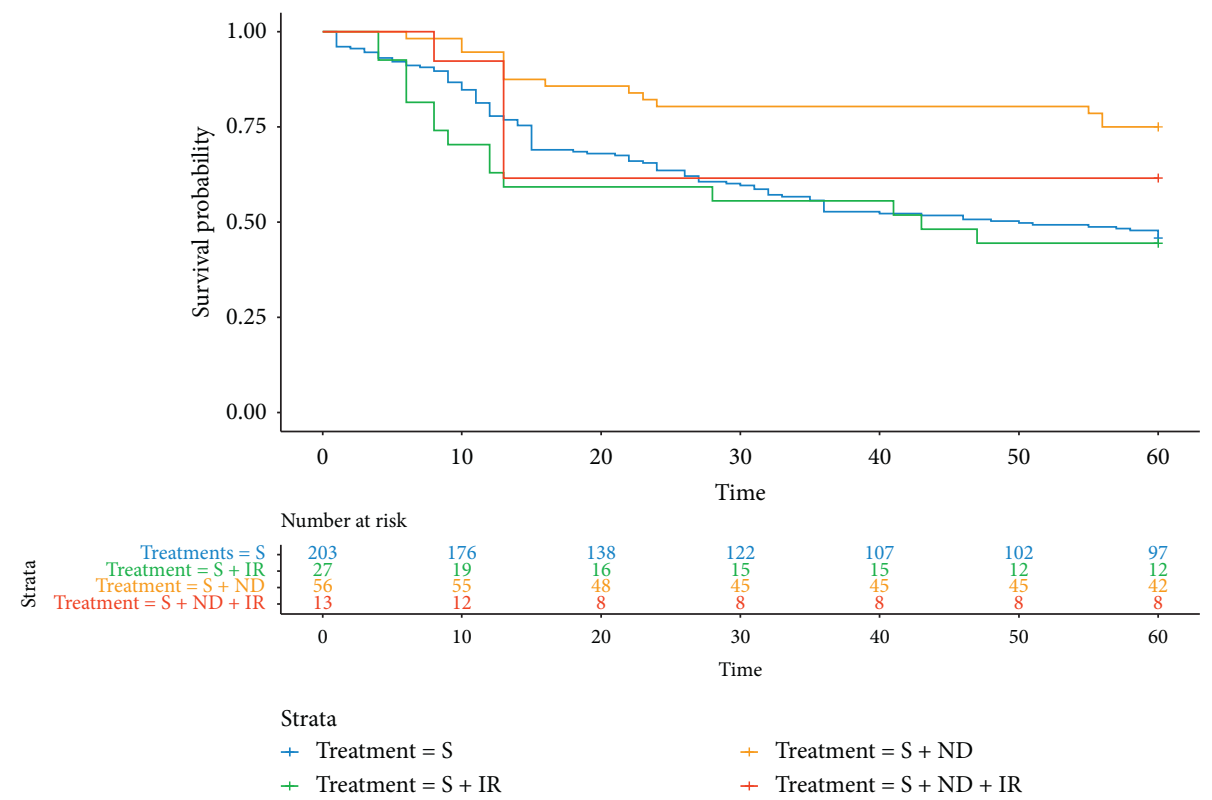

(a)

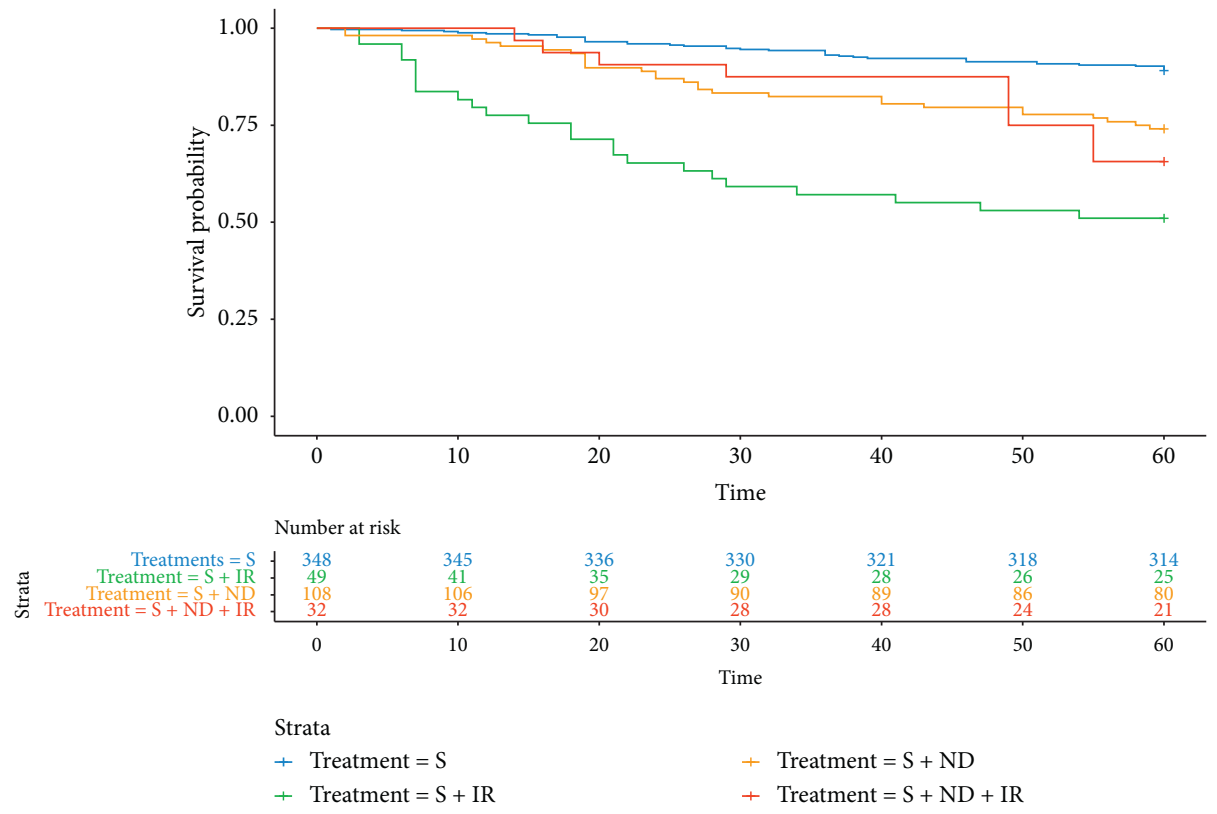

(b)

FIGURE 1: (a) Kaplan-Meier curve demonstrating survival benefit of surgery with neck dissection over other treatment modalities in oral squamous cell carcinoma patients with high mortality risk scores $(\geq 0)$. ${ }^{*}$ Time in months. (b) Kaplan-Meier curve delineating survival benefit of surgery only over other treatment modalities in oral squamous cell carcinoma patients with low mortality risk scores $(<0) .{ }^{*}$ Time in months.

$4736,655-3 p$ [7]. In this study, we assessed the intersection among these genes that control cancer recurrence and aggressiveness (Figure 2). The Rac family small GTPase 1 (Rac1) and the Rho GTPases activated p21-activated kinases (PAKs) pathway, including Cdc42 and hTERT, were identified as key targets of the miRNAs. Rho GTPases are crucial regulators of cell migration and are altered in many cancer types, including colon, glioblastoma, and head and neck cancer [15]. Rac, Rho, and Cdc42 are subfamilies of Rho GTPase [16]. Integrin-mediated cell-extracellular matrix adhesion activates Rac1, which directly binds and activates PAKs and other effectors including phosphatidylinositol-4phosphate 5-kinase, Nap125, PIR121, and IRSp53, resulting in increased membrane protrusions and actin polymerization $[16,17]$. The other member of Rho GTPase subfamily Cdc42, once activated, phosphorylates PAK1 and PAK2, which in turn leads to filopodia formation [17]. These changes either by themselves or integrated can affect gene expression, cell cycle progression, and apoptosis [17]. Importantly, Cdc42/Rac1 participates in the 
TABLE 3: The median time-to-recurrence, time-to-death, and survival rate in oral squamous cell carcinoma patients treated with surgery only $(S)$ or surgery with neck dissection $(S+N D)$. Initial high- versus low-risk stratification using 0 as cutoff, and then subrisk stratification into highest, moderately high, moderately low, and low risks are shown. For clinical use, the patients can be categorized into high versus moderate versus low-risk groups; the "high-risk strata $(\geq 2)$ " represents a group of patients at greater risk of cancer relapse and death even after $S+\mathrm{ND}$, compared to "moderate-risk strata $(0$ to $<2)$ ", in which survival greatly improves with $S+$ ND. For the "low-risk strata $(<0)$ ", $S$ is associated with survival benefit.

\begin{tabular}{|c|c|c|c|c|c|c|c|}
\hline $\begin{array}{l}\text { Risk } \\
\text { strata }\end{array}$ & Sub-risk strata & $\begin{array}{l}\text { Risk } \\
\text { scores }\end{array}$ & $\begin{array}{l}\text { Median time-to- } \\
\text { recurrence }\end{array}$ & $\begin{array}{l}\text { Median time-to- } \\
\text { death }\end{array}$ & $\begin{array}{c}\text { Cancer death } \\
(\%)\end{array}$ & $\begin{array}{c}\text { 5-year survival } \\
(\%)\end{array}$ & $\begin{array}{c}\text { Clinical } \\
\text { risks }\end{array}$ \\
\hline \multirow{9}{*}{$\begin{array}{l}\text { High- } \\
\text { risk }\end{array}$} & Highest risk & & & & & & \multirow{6}{*}{ High-risk } \\
\hline & S Only & $\geq 2$ & 6 (months) & 11 (months) & 94 & 6 & \\
\hline & $\mathrm{S}+\mathrm{ND}$ & & 58 & $\geq 60$ & 32 & 68 & \\
\hline & Moderately high risk & \multirow{3}{*}{1 to $<2$} & & & & & \\
\hline & S only & & 14 & 22 & 71 & 29 & \\
\hline & $\mathrm{S}+\mathrm{ND}$ & & $\geq 60$ & $\geq 60$ & 0 & 100 & \\
\hline & Moderately low risk & \multirow{3}{*}{0 to $<1$} & & & & & \multirow{3}{*}{$\begin{array}{l}\text { Moderate- } \\
\text { risk }\end{array}$} \\
\hline & S Only & & 46 & $\geq 60$ & 42 & 58 & \\
\hline & $\mathrm{S}+\mathrm{ND}$ & & $\geq 60$ & $\geq 60$ & 0 & 100 & \\
\hline \multirow{3}{*}{ Low-risk } & Low risk & \multirow{3}{*}{$<0$} & & & & & \multirow{3}{*}{ Low-risk } \\
\hline & S Only & & $\geq 60$ & $\geq 60$ & 11 & 89 & \\
\hline & $\mathrm{S}+\mathrm{ND}$ & & $\geq 60$ & $\geq 60$ & 26 & 74 & \\
\hline
\end{tabular}

posttranscriptional control of telomerase activity by transcriptional upregulation of human telomerase reverse transcriptase (hTERT) [18, 19]. Hence, PAKs/Rac1-Cdc42hTERT signaling pathway may play a central role in cancer recurrence and aggressiveness.

3.5. Expression Level Analysis of Rac1, Cdc42, and hTERT by $q R T-P C R$. We assessed expression levels of Rac1, Cdc42, and hTERT using qRT-PCR. Due to the shortage of samples and materials, the expression levels of three gene targets were studied in forty-one patients who had S or $S+$ ND. Gene expression levels were also assessed in oral tissue samples from five healthy individuals for comparison. cDNA was made from total RNA using high-capacity cDNA Reverse Transcription Kit (Applied Biosystems, USA) according to the manufacturer's protocol. The expression levels were measured by qRT-PCR (LightCycler 480 II, Roche Applied Science). The following TaqMan Gene Expression Assays were used (Applied Biosystems): hTERT (Hs00972650_m1), Cdc42 (Hs0198044_g1), and Rac1 (Hs01902432_s1). The reactions were carried out in a total volume of $12.5 \mu \mathrm{l}$, with $1 \mathrm{X}$ power TaqMan Fast Advanced Master Mix and $0.2 \mu \mathrm{M}$ of each primer. Data was analyzed to determine the threshold cycle (Ct). The expression levels were normalized using GAPDH as an endogenous control $(\Delta \mathrm{Ct})$. The $\Delta \Delta \mathrm{Ct}$ and fold change in expression levels calculated for each sub-risk strata compared to the healthy controls are shown in Table 4.

hTERT expression levels were mostly undetectable in the control samples, and Ct of 39.99 was used. hTERT demonstrated 22-fold increase in the highest-risk substrata compared to healthy controls $(p<0.0005)$. Racl and Cdc42 were also overexpressed in the highest-risk substrata, although statistical significance was not reached. The expression levels of three gene targets correlated with the risk scores, demonstrating that elevated hTERT, Rac1, and Cdc42 levels are associated with cancer recurrence and poor prognosis.
3.6. hTERT Expression by Quantitative Immunofluorescent Assay. To further assess hTERT expression in more aggressive form of OSCC, we performed quantitative immunofluorescent assays in ten patients with risk scores of 2 or higher who had cancer recurrence. The formalin-fixed paraffin-embedded tissue slides were stained with anti-human hTERT (Rockland Immunochemicals, Limerick, PA). The hTERT stain concentration and the number of nuclei staining with hTERT were quantified using a macro derived from the Leica Quantitative Algorithm v1. The hTERT stain concentration was multiplied by the number of hTERTexpressing tumor cells to obtain final hTERT expression levels. After adjusting for the outliers, we had eight evaluable cases. Differential hTERT expression levels were compared between those who had cancer-specific death following recurrence $(n=4)$ vs those who had 5-year survival despite having a recurrence $(n=4)$ (Figure $3(\mathrm{a})$ ). hTERT levels were 2.4-fold higher in those who had a cancer-specific death (an average hTERT expression level of 8,061; an average hTERT stain concentration of 3.8, with an average of 2,318 hTERTexpressing tumor cells) compared to those with 5-year survival (an average hTERT expression level of 3,330; an average hTERT stain concentration of 3.5, with an average of 884 hTERT-expressing tumor cells). hTERT level correlated with the mortality risk scores, demonstrating that elevated hTERT level is associated with poor survival.

We also assessed feasibility of hTERT level quantification in the patient-derived normal and OSCC organoids. We first obtained small piece $(5 \times 5 \times 3 \mathrm{~mm})$ of normal and OSCC tissue from patients at the time of surgery. Surface epithelium from normal tissues and tumor islands within lamina propria from OSCC tissues were isolated. Tissue sections were minced, enzymatically dissociated, and forced through a $70 \mu \mathrm{m}$ cell strainer. The single cell suspension $\left(4 \times 10^{5} / \mathrm{ml}\right)$ was mixed with Matrigel (Corning, Corning, NY) and $2 \times 10^{4}$ cells in $50 \mu \mathrm{l}$ of Matrigel were seeded into the 24-well plates. Organoids were grown for $10-14$ days at $37^{\circ} \mathrm{C}$ in a humidified atmosphere of $5 \% \mathrm{CO}_{2}$ as previously described [19]. Under an inverted 


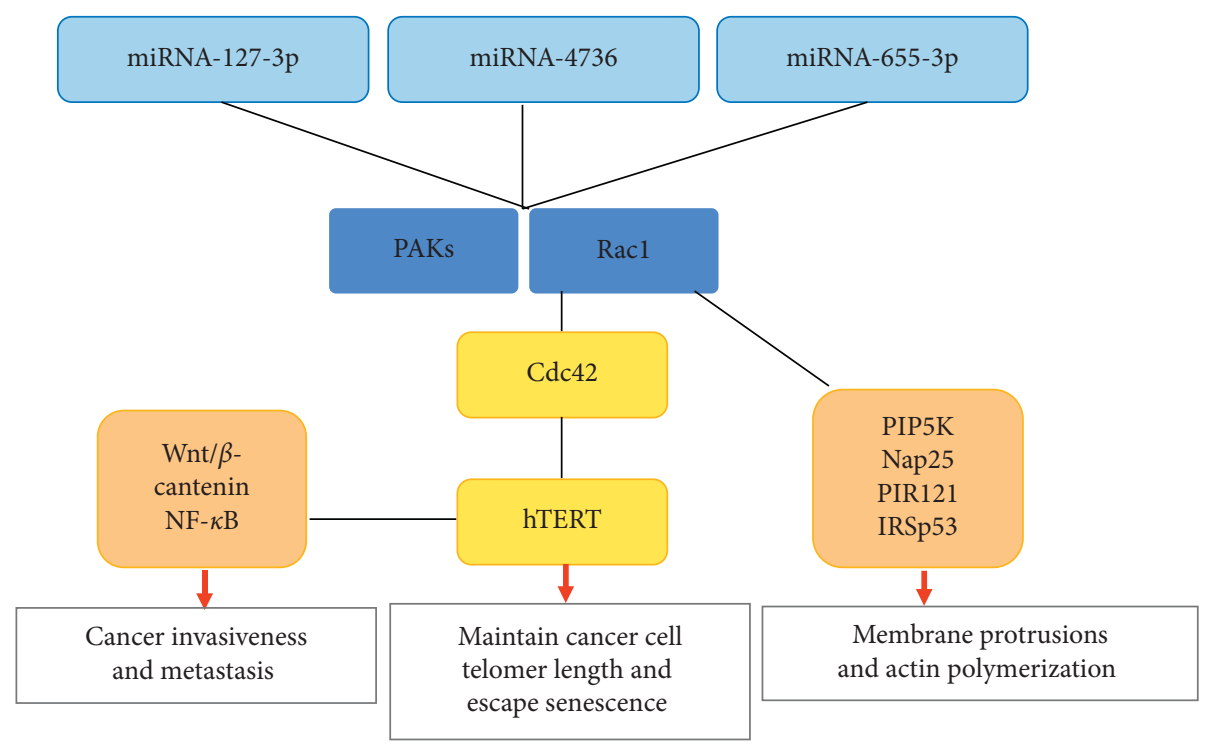

(a)

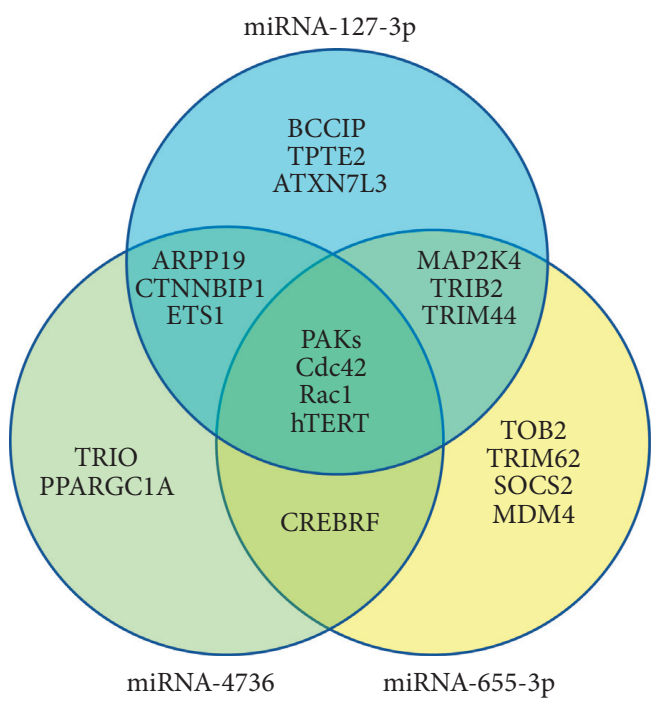

(b)

Figure 2: Common gene targets of microRNA-127-3p, 4736, 655-3p that are associated with cancer recurrence and aggressiveness. (a) The cancer-related pathway involving Rac family small GTPase 1 (Rac1), p21-activated kinases (PAKs), Cdc42, and human telomerase reverse transcriptase (hTERT) was targeted by three microRNAs. (b) Venn diagram showing common gene targets of three microRNAs. Gene targets. Venn diagram.

TABLE 4: The $\Delta \Delta \mathrm{Ct}$ and the fold change in the expression levels of Rac1, Cdc42, and hTERT in four sub-risk strata compared to healthy controls. The hTERT expression levels significantly correlated with the risk scores.

\begin{tabular}{|c|c|c|c|c|c|c|c|}
\hline \multirow{2}{*}{ Sub-risk strata } & \multirow{2}{*}{ Risk score } & \multicolumn{3}{|c|}{$\Delta \Delta \mathrm{Ct}$} & \multicolumn{3}{|c|}{ Fold change } \\
\hline & & Rac1 & $\mathrm{Cdc} 42$ & hTERT & Rac1 & Cdc42 & hTERT \\
\hline Highest risk & $\geq 2$ & 3.22 & 1.05 & $4.55^{*}$ & 8.32 & 1.07 & $22.43^{*}$ \\
\hline Moderately high risk & 1 to $<2$ & 2.95 & 0.88 & $3.86^{*}$ & 6.71 & 0.84 & $13.53^{*}$ \\
\hline Moderately low risk & 0 to $<1$ & 2.77 & 0.23 & $3.61^{*}$ & 5.81 & 0.17 & $11.17^{*}$ \\
\hline Low risk & $<0$ & 2.00 & 0.21 & $3.42^{*}$ & 3.01 & 0.16 & $9.78^{*}$ \\
\hline Healthy controls & - & Reference & Reference & Reference & Reference & Reference & Reference \\
\hline
\end{tabular}

${ }^{*} p<0.05$ when compared with the healthy controls.

phase-contrast microscope, growing organoids were observed and photomicrographed to determine their number and size. Organoid formation rate was defined as the average number of $\geq 50 \mu \mathrm{m}$ spherical structures at day 14 divided by the total number of cells seeded in each well at day 0 . Organoids were recovered and fixed overnight in paraformaldehyde for 

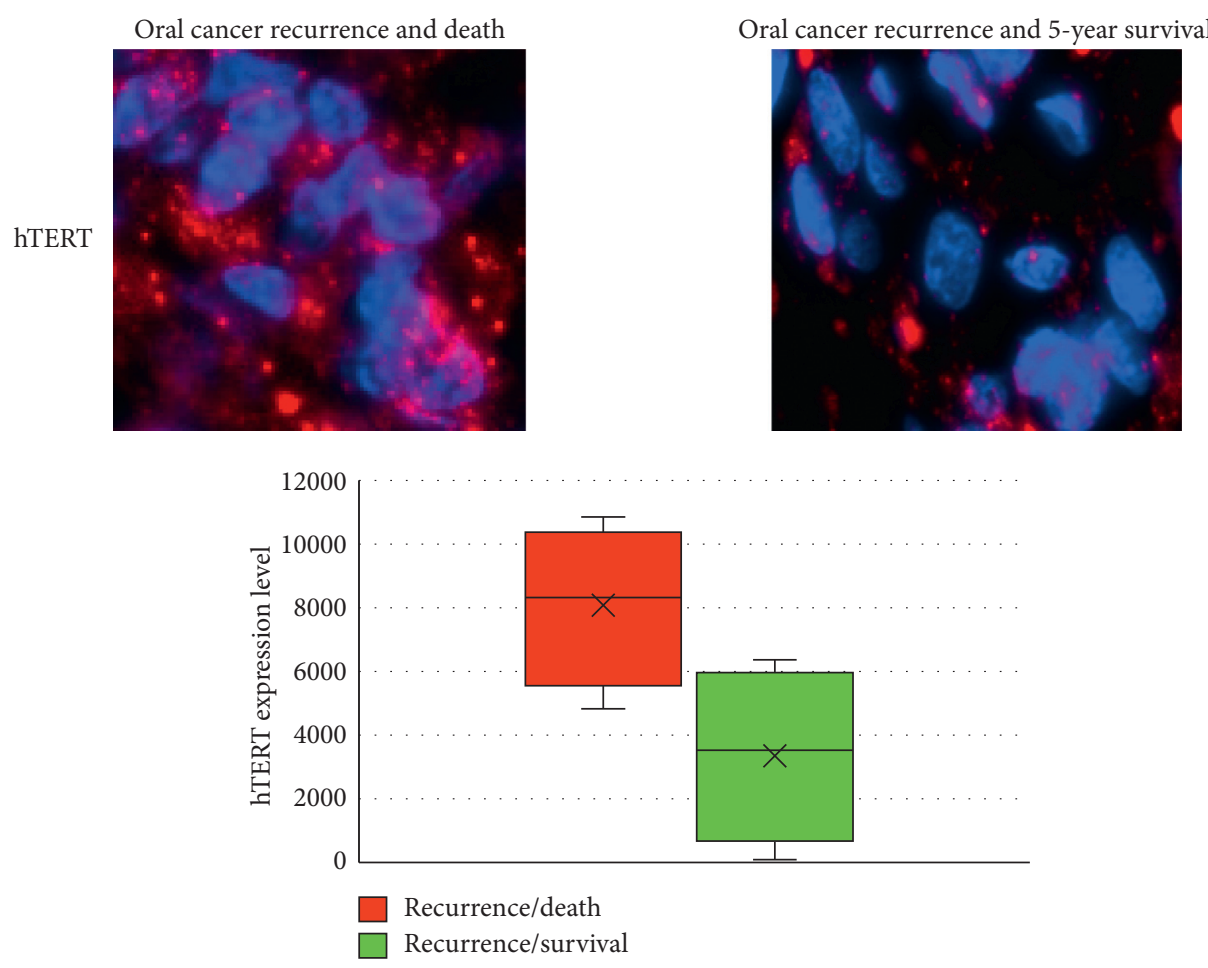

(a)
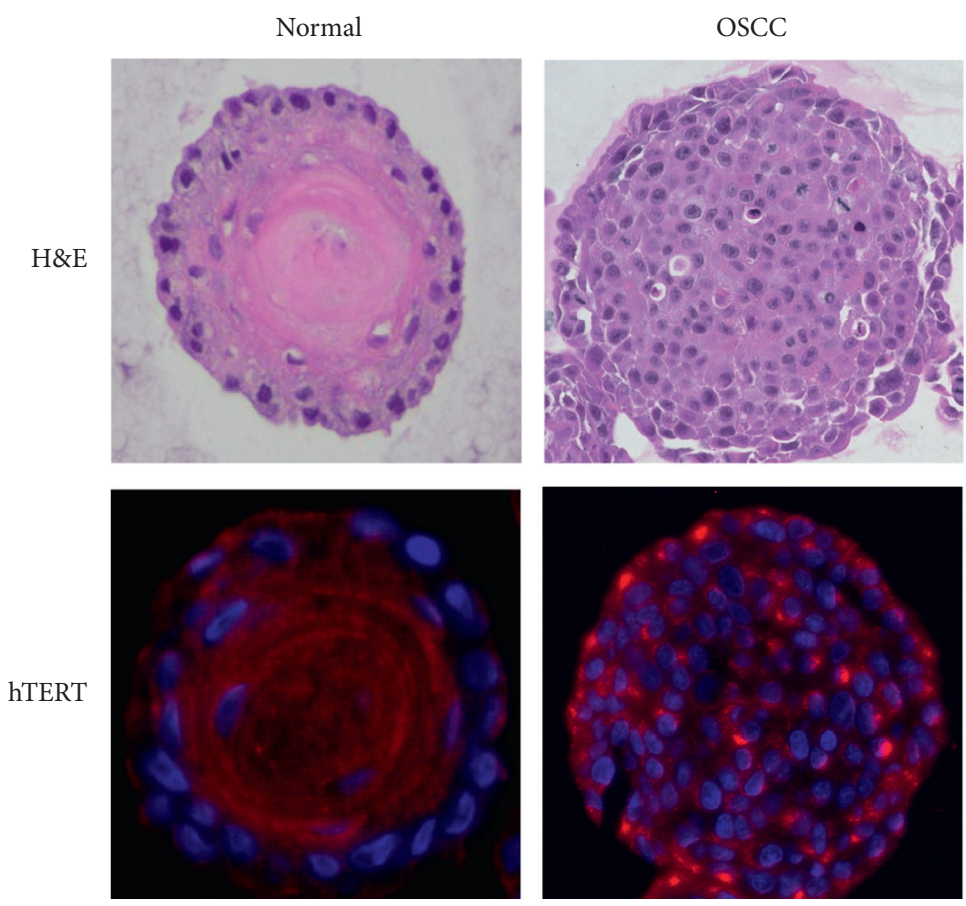

(b)

FIgURE 3: The hTERT expression levels assessed by quantitative immunofluorescence assay. (a) Elevated hTERT expression levels (bright red dots on blue DAPI-stained nuclei) were observed in patients with poor prognosis (cancer recurrence and death within 5-year period) compared to those who survived the 5-year period despite having cancer recurrence. Dot plots comparing hTERT expression levels in poor versus favorable prognostic group. Bars indicate the mean hTERT expression level. (b) Minimal hTERT expression level was detected in normal oral organoids. In comparison, elevated hTERT expression level (bright red nuclear and peri-nuclear dots) was observed in oral squamous cell carcinoma (OSCC) organoids. 
histological examination and hTERT quantitative immunofluorescent assay. There were minimal hTERT immunofluorescent staining in normal organoids and stronger immunofluorescent staining in OSCC organoids (Figure 3(b)).

\section{Discussion}

Currently there is no clinical modality to identify patients at high risk for cancer-specific death among those assigned to early-stage OSCC (TNM stages I and II). Because $80 \%$ of oral cancer patients are in early stage at the time of diagnosis [2], a window of opportunity exists in which proper prognostication and subsequent decisions for additional treatment can dramatically improve the 5-year survival of patients with this deadly disease.

Using the miRNA-based mortality risk score formula, we assigned a risk score to all patients and assessed the survival benefit in high $(\geq 0)$ vs. low $(<0)$ risk strata based on the treatment received. Compared to $\mathrm{S}, S+\mathrm{ND}$ significantly increased the median time-to-recurrence from 26 months to $\geq 60$ months and the 5-year survival rate from $46 \%$ to $75 \%$ $(p<0.001)$ for the high-risk group, consistent with other reports that $S+\mathrm{ND}$ is associated with survival benefit in early-stage OSCC $[20,21]$. Improved 5-year survival in the $S+$ ND group was most likely secondary to prolonged timeto-recurrence. Indeed, initial treatment modality and timeto-recurrence were reported to be independent prognostic variables [22].

Paradoxically, the patients in the low mortality risk group who received $S+\mathrm{ND}$ had a lower 5-year survival rate of $74 \%$ compared to $89 \%$ in the $S$ only group. The significant reduction in the 5-year survival in the low-risk strata who had $S+$ ND may be explained by the low success rate of salvage surgery at the time of cancer recurrence [22, 23]. With initial $S+$ ND treatment, patients experienced recurrence in a less predictable fashion, often involving the contralateral neck $[22,23]$. In contrast, patients treated with S only were more likely to present with nodal disease confined to the neck at levels I, II, and III, which were successfully removed during salvage surgery [22, 23]. Consistently, of the high-risk patients in our study who were initially treated with $S+\mathrm{ND}, 54 \%$ experienced cancer recurrence and died of disease due to failed salvage surgery. $S+\mathrm{IR}$ and $S+\mathrm{IR}+\mathrm{ND}$ demonstrated minimal survival benefit over $S$ or $S+$ ND for both high- and low-risk strata.

Based on the assessment of sub-risk strata [highest $(\geq 2)$, moderately high $(<2-1)$, moderately low $(<1-0)$, and low $(<0)$ ], it may be beneficial for patients to be risk stratified using the prognostic marker at the time of biopsy. For those with low mortality risk scores ( $<0$; low clinical risk), surgery only without neck dissection is the most beneficial treatment modality. On the other hand, for those with risk scores between 0 and $\leq 1$ (moderate clinical risk), $S+$ ND instead of $S$ will improve the 5 -year survival rate to $100 \%$. However, for the patients with the highest-risk score of $\geq 2$ (high clinical risk), while $S+\mathrm{ND}$ improves the 5-year survival rate over $\mathrm{S}$ to $68 \%$, it is still suboptimal. These patients may benefit from additional therapy following surgery to prevent cancer recurrence, which will further improve the 5-survival rate.
Human telomerase reverse transcriptase (hTERT) along with few other molecules in the same signaling pathway were demonstrated to be targeted by all three miRNAs. Indeed, there was 22-fold increase in the hTERT expression levels in the highest-risk substrata compared to healthy controls $(p<0.0005)$. Consistently, our quantitative immunofluorescence study demonstrated elevated levels of hTERT in OSCC patients who had cancer recurrence and death, compared to those who survived despite having cancer relapse. In patient-derived organoids, we showed that there is overexpression of hTERT in OSCC and minimal expression in normal organoids. Others have reported similar findings. Elevated expression of hTERT was associated with a poor prognosis in solid tumors such as gastric, lung, cervical, head and neck, breast, and ovarian cancer, and glioblastoma [24, 25]. In head and neck cancer patients, elevated hTERT expression was associated with a higher recurrence rate $(p=0.044)$ and a lower 5-year survival rate $(p=0.011)$ [26]. An elevated level of hTERT was also observed in oral cells within the cancerized field [27]. Moreover, hTERT expression level correlated with degree of oral preneoplasia [27]. Compared to normal oral mucosa, hTERT expression was elevated by a 6.9-fold in OSCC [28]. Thus, the hTERT signaling axis is a viable therapeutic target for oral cancer patients.

Three-dimensional (3D) tumor organoid models have been consistently shown to faithfully recapitulate features of the tumor of origin in terms of cell differentiation, heterogeneity, histoarchitecture, and clinical drug response [29-32]. Thus, there is increasing interest in developing tumor organoid models for drug development and personalized medicine applications. Functional precision therapy approaches where the primary tumor tissue is directly exposed to drugs to determine efficacy have the potential to boost personalized medicine efforts and influence clinical decisions [33-36]. A recent study found that patientderived organoids could accurately predict patient responses to therapy, with $100 \%$ sensitivity and $93 \%$ specificity [37]. While establishing patient-derived xenografts is costly and time-consuming, in vitro $3 \mathrm{D}$ organoids derived from primary cancers can be established rapidly, with successful passage within 10-14 days of in vitro growth and with $>80 \%$ efficiency [29]. Thus far, we have cultivated patient-derived organoids from normal oral epithelium and OSCC. These organoids can be utilized in the future as a functional in vitro testing platform to explore novel therapeutic options, which has a potential role in clinical decision-making tailored to each individual.

\section{Conclusion}

In response to the critical need to subdivide traditional tumor classes into subsets that behave differently and also to refine and improve prognostication and treatment selection, we have developed and validated a novel miRNA-based prognostic model to predict survival outcome in patients who are already categorized into "early-stage" by the TNM system. For clinical practicality, we developed a parsimonious risk score formula that is capable of translating 
miRNA expression levels assessed by qRT-PCR at the time of initial cancer diagnosis into a score that reflects the risk of cancer-specific mortality during the 5-year period from the time of surgery. Improved survival was observed in the highrisk strata who received $S+\mathrm{ND}$ and in the low-risk strata receiving $S$ as the initial treatment. Moreover, we demonstrated that for patients with risk scores of $\geq 2$, there is suboptimal increase of 5-year survival to $68 \%$ with $S+$ ND. Hence, these patients may benefit from therapeutic intervention in addition to surgical treatment to further improve survival. We also demonstrated that hTERT overexpression is associated with shorter median time-to-recurrence and death, making it a promising drug target. Cancer vaccines that prime immune system against hTERT-expressing cells are currently undergoing clinical trials for various solid tumors [38-40]. If delivered following surgical treatment, these vaccines can be repurposed to prevent cancer recurrence and improve survival in high-risk early-stage oral cancer patients.

\section{Data Availability}

The data provided in this publication will be available from the corresponding author upon request.

\section{Conflicts of Interest}

The authors declare that they have no conflicts of interest regarding the publication of this paper.

\section{Acknowledgments}

The authors thank Charis Yoon for her assistance in data organization and Qiao Wang for her technical assistance. This work was supported by the NIH/NIDCR R01DE026801 (A. Yoon), the NIH/NIEHS P30ES009089 and the NIH/NCI P30CA013696, (Columbia University), NIH/NCI P01CA098101 (H. Nakagawa), NIH/NCI U54CA163004 (H. Nakagawa), NIH/ NIAAA R01AA026297 (H. Nakagawa), the Translational Research Program at WCMC Pathology and Laboratory Medicine, the NIH/NCI P30CA086862 (University of Iowa), and the NIH/ NCI P30CA071789 (University of Hawaii Cancer Center).

\section{References}

[1] C. Rivera, "Essentials of oral cancer," International Journal of Clinical and Experimental Pathology, vol. 8, no. 9, pp. 11884-11894, 2015.

[2] T. Sasahira and T. Kirita, "Hallmarks of cancer-related newly prognostic factors of oral squamous cell carcinoma," International Journal of Molecular Sciences, vol. 19, no. 8, p. 2413, 2018.

[3] F. R. Pires, A. B. Ramos, J. B. C. D. Oliveira, A. S. Tavares, P. S. R. D. Luz, and T. C. R. B. D. Santos, "Oral squamous cell carcinoma: clinicopathological features from 346 cases from a single oral pathology service during an 8-year period," Journal of Applied Oral Science, vol. 21, no. 5, pp. 460-467, 2013.

[4] D. J. Weatherspoon, A. Chattopadhyay, S. Boroumand, and I. Garcia, "Oral cavity and oropharyngeal cancer incidence trends and disparities in the United States: 2000-2010," Cancer Epidemiology, vol. 39, no. 4, pp. 497-504, 2015.
[5] P. M. De Abre, A. C. G. Co, and P. L. Azevedo, "Frequency of HPV in oral cavity squamous cell carcinoma," BMC Cancer, vol. 18, p. 324, 2018.

[6] W. M. Lydiatt, S. G. Patel, B. O’Sullivan et al., "Head and neck cancers-major changes in the American joint committee on cancer eighth edition cancer staging manual," CA: A Cancer Journal for Clinicians, vol. 67, no. 2, pp. 122-137, 2017.

[7] A. J. Yoon, S. Wang, D. I. Kutler et al., "MicroRNA-based risk scoring system to identify early-stage oral squamous cell carcinoma patients at high-risk for cancer-specific mortality," Head \& Neck, vol. 42, no. 8, pp. 1699-1712, 2020.

[8] K. Gombos, R. Horváth, E. Szele et al., "miRNA expression profiles of oral squamous cell carcinomas," Anticancer Research, vol. 33, no. 4, pp. 1511-1517, 2013.

[9] G. Troiano, F. Mastrangelo, V. C. A. Caponio, L. Laino, N. Cirillo, and L. Lo Muzio, "Predictive prognostic value of tissue-based MicroRNA expression in oral squamous cell carcinoma: a systematic review and meta-analysis," Journal of Dental Research, vol. 97, no. 7, pp. 759-766, 2018.

[10] G. S. Sarode, S. C. Sarode, N. Maniyar, R. Anand, and S. Patil, "Oral cancer databases: a comprehensive review," Journal of Oral Pathology \& Medicine, vol. 47, no. 6, pp. 547-556, 2018.

[11] J. Myers, "Potential role of micro-RNAs in head and neck tumorigenesis," Head \& Neck, vol. 32, pp. 1099-1011, 2010.

[12] M. Manikandan, A. K. Deva Magendhra Rao, and G. Arunkumar, "Oral squamous cell carcinoma: microRNA expression profiling and integrative analyses for elucidation of tumourigenesis mechanism," Molecular Cancer, vol. 15, p. 28, 2016.

[13] L. Ramdas, U. Giri, C. L. Ashorn et al., "MiRNA expression profiles in head and neck squamous cell carcinoma and adjacent normal tissue," Head \& Neck, vol. 31, no. 5, pp. 642-654, 2009.

[14] C. Kumarasamy, M. R. Madhav, S. Sabarimurugan et al., "Prognostic value of miRNAs in head and neck cancers: a comprehensive systematic and meta-analysis," Cells, vol. 8, no. 8, p. 772, 2019.

[15] Y. Lin and Y. Zheng, "Approaches of targeting Rho GTPases in cancer drug discovery," Expert Opinion on Drug Discovery, vol. 10, no. 9, pp. 991-1010, 2015.

[16] G. A. Cardama, N. Gonzalez, J. Maggio, P. L. Menna, and D. E. Gomez, "Rho GTPases as therapeutic targets in cancer (Review)," International Journal of Oncology, vol. 51, no. 4, pp. 1025-1034, 2017.

[17] E. Sahai and C. J. Marshall, "RHO-GTPases and cancer," Nature Reviews Cancer, vol. 2, no. 2, pp. 133-142, 2002.

[18] D. L. Gomez, R. G. Armando, C. S. Cerrudo, P. D. Ghiringhelli, and D. E. Gomez, "Telomerase as a cancer target. development of new molecules," Current Topics in Medicinal Chemistry, vol. 16, no. 22, pp. 2432-2440, 2016.

[19] Y. M. Yeh, Y. T. Pan, and T.C. V. Wang, "Cdc42/Rac1 participates in the control of telomerase activity in human nasopharyngeal cancer cells," Cancer Letters, vol. 218, no. 2, pp. 207-213, 2005.

[20] D. Mirea, R. Grigore, D. Safta et al., "Elective neck dissection in patients with stage T1-T2N0 carcionma of the anterior tongue," Hippokratia, vol. 18, pp. 120-124, 2014.

[21] A. Mizrachi, J. C. Migliacci, P. H. Montero et al., "Neck recurrence in clinically node-negative oral cancer: 27-year experience at a single institution," Oral Oncology, vol. 78, pp. 94-101, 2018.

[22] M. D. Kernohan, J. R. Clark, K. Gao, A. Ebrahimi, and C. G. Milross, "Predicting the prognosis of oral squamous cell 
carcinoma after first recurrence," Archives of OtolaryngologyHead \& Neck Surgery, vol. 136, no. 12, pp. 1235-1239, 2010.

[23] L. P. Kowalski, "Results of salvage treatment of the neck in patients with oral cancer," Archives of Otolaryngology-Head \& Neck Surgery, vol. 128, no. 1, pp. 58-62, 2002.

[24] K. Wang, R. L. Wang, and J. J. Liu, "The prognostic significance of hTERT overexpression in cancers: a systemic review and meta-analysis," Medicine, vol. 98, Article ID e11794, 2018.

[25] K. L. Kirkpatrick and K. Mokbel, "The significance of human telomerase reverse transcriptase (hTERT) in cancer," European Journal of Surgical Oncology (EJSO), vol. 27, no. 8, pp. 754-760, 2001.

[26] H. H. Che, C. H. Yu, and J. T. Wang, "Expression of human telomerase reverse transcriptase (hTERT) protein is significantly associated with the progression, recurrence and prognosis of oral squamous cell carcinoma in Taiwan," Oral Oncology, vol. 43, pp. 122-129, 2007.

[27] H. R. Kim, R. Christensen, N. H. Park, P. Sapp, M. K. Kang, and N. H. Park, "Elevated expression of hTERT is associated with dysplastic cell transformation during human oral carcinogenesis in situ," Clinical Cancer Research:An Official Journal of the American Association for Cancer Research, vol. 7, no. 10, pp. 3079-3086, 2001.

[28] E. M. Fabricius, U. Gurr, and G. P. Wildner, "Telomerase activity levels in the surgical margin and tumour distant tissue of the squamous cell carcinoma of the head-and-neck," Analytical Cellular Pathology, vol. 24, no. 1, pp. 25-39, 2002.

[29] T. A. Karakasheva, T. Kijima, M. Shimonosono et al., "Generation and characterization of patient-derived head and neck, oral, and esophageal cancer organoids," Current Protocols in Stem Cell Biology, vol. 53, no. 1, p. e109, 2020.

[30] E. Driehuis, S. Kolders, S. Spelier et al., "Oral mucosal organoids as a potential platform for personalized cancer therapy," Cancer Discovery, vol. 9, no. 7, pp. 852-871, 2019.

[31] T. Kijima, H. Nakagawa, M. Shimonosono et al., "Three-dimensional organoids reveal therapy resistance of esophageal and oropharyngeal squamous cell carcinoma cells," Cellular and Molecular Gastroenterology and Hepatology, vol. 7, no. 1, pp. 73-91, 2019.

[32] N. Phan, J. J. Hong, and B. Tofig, “A simple high-throughput approach identifies actionable drug sensitivities in patientderived tumor organoids," Communications Biology, vol. 2, p. 78, 2019.

[33] C. Cummings, E. Peters, L. Lacroix, F. Andre, and M. Lackner, "The role of next-generation sequencing in enabling personalized oncology therapy," Clinical and Translational Science, vol. 9, no. 6, pp. 283-292, 2016.

[34] R. Simon and S. Roychowdhury, "Implementing personalized cancer genomics in clinical trials," Nature Reviews Drug Discovery, vol. 12, no. 5, pp. 358-369, 2013.

[35] A. Letai, "Functional precision cancer medicine-moving beyond pure genomics," Nature Medicine, vol. 23, no. 9, pp. 1028-1035, 2017.

[36] G. Vlachogiannis, S. Hedayat, A. Vatsiou et al., "Patientderived organoids model treatment response of metastatic gastrointestinal cancers," Science, vol. 359, no. 6378, pp. 920-926, 2018.

[37] R. D. Gartrell, D. K. Marks, T. D. Hart et al., "Quantitative analysis of immune infiltrates in primary melanoma," Cancer Immunology Research, vol. 6, no. 4, pp. 481-493, 2018.

[38] C. C. Lin, "Should we design clinical trials differently in the era of cancer immunotherapy?" Journal of Immunotherapy and Precision Oncology, vol. 2, no. 2, pp. 36-39, 2019.
[39] J. Yan, P. Pankhong, T. H. Shin et al., "Highly optimized DNA vaccine targeting human telomerase reverse transcriptase stimulates potent antitumor immunity," Cancer Immunology Research, vol. 1, no. 3, pp. 179-189, 2013.

[40] I. Melero, A. M. Grimaldi, J. L. Perez-Gracia, and P. A. Ascierto, "Clinical development of immunostimulatory monoclonal antibodies and opportunities for combination," Clinical Cancer Research, vol. 19, no. 5, pp. 997-1008, 2013. 